\title{
Constraints on sequence processing speed in biological neuronal networks
}

\author{
Younes Bouhadjar ${ }^{1,2}$, Markus Diesmann ${ }^{1,3}$, Rainer Waser $^{2,4}$, Dirk J. Wouters ${ }^{4}$, Tom Tetzlaff ${ }^{1}$ \\ ${ }^{1}$ Institute of Neuroscience and Medicine (INM-6) \& Institute for Advanced Simulation (IAS-6) \& JARA Institute Brain Structure-Function Relationships \\ (INM-10), Jülich Research Centre, Jülich, Germany \\ 2 Peter Grünberg Institute (PGI-7), Jülich Research Centre and JARA, Jülich, Germany \\ ${ }^{3}$ Department of Psychiatry, Psychotherapy and Psychosomatics, Medical Faculty \& Department of Physics, Faculty 1, RWTH Aachen University, \\ Aachen, Germany \\ ${ }^{4}$ Institute of Electronic Materials (IWE 2) \& JARA-FIT, RWTH Aachen University, Aachen, Germany
}

\begin{abstract}
Sequence processing has been proposed to be the universal computation performed by the neocortex. The Hierarchical Temporal Memory (HTM) model provides a mechanistic implementation of this form of processing. While the model accounts for a number of neocortical features, it is based on networks of highly abstract neuron and synapse models updated in discrete time. Here, we reformulate the model in terms of a network of spiking neurons with continuous-time dynamics to investigate how neuronal parameters such as cell-intrinsic time constants and synaptic weights constrain the sequence-processing speed.
\end{abstract}

\section{CCS CONCEPTS}

- Networks $\rightarrow$ Network dynamics; • Computing methodologies $\rightarrow$ Massively parallel algorithms; Temporal reasoning.

\section{KEYWORDS}

sequence processing, neocortex, hierarchical temporal memory, spiking neurons, dendritic action potentials, spike timing dependent structural plasticity

\section{INTRODUCTION}

Learning and processing temporal sequences have been suggested to be the fundamental computation performed by the neocortex [13]. The Hierarchical Temporal Memory (HTM) model constitutes a mechanistic description of this type of computation [4]. It accounts for the specific anatomical structure of cortical (pyramidal) neurons, explains the functional role of dendritic action potentials, and learns continuously by means of local learning rules. The model can simultaneously learn and predict multiple sequences in streams of data and is robust with respect to failure of network elements and noise. So far, implementations of this model are based on highly abstract models of neurons and synapses with discrete-time dynamics. To foster an understanding of the sequence processing characteristics

Permission to make digital or hard copies of all or part of this work for personal or classroom use is granted without fee provided that copies are not made or distributed for profit or commercial advantage and that copies bear this notice and the full citation on the first page. Copyrights for components of this work owned by others than the author(s) must be honored. Abstracting with credit is permitted. To copy otherwise, or republish, to post on servers or to redistribute to lists, requires prior specific permission and/or a fee. Request permissions from permissions@acm.org.

ICONS '19, July 23-25, 2019, Knoxville, TN, USA

(c) 2019 Copyright held by the owner/author(s). Publication rights licensed to ACM. ACM ISBN 978-1-4503-7680-8/19/07 ..\$15.00

https://doi.org/10.1145/3354265.3354281 in humans and other mammals, the model needs to be reformulated in terms of biophysical principles and parameters.

In this study, we deliver a continuous-time implementation of the temporal-memory algorithm proposed by the HTM theory [5], which comprises networks of spiking neurons, dendritic action potentials, backpropagating action potentials, lateral inhibition, and spike timing dependent structural plasticity. In the framework of this model, we investigate to what extent the sequence processing speed is constrained by neuronal parameters such as cell-intrinsic time constants and synaptic weights. We test the implementation in a task where the network learns random sequences of letters, and study the role of the inter-stimulus interval on the sequence prediction error, thereby deriving lower and upper bounds for the sequence processing speed.

\section{MODEL}

In the following, we will provide an overview of the network model components. A detailed description of the model and parameter values can be found in Appendix A and B.

\section{$2.1 \quad$ Network model}

The network consists of $M$ minicolumns. Each minicolumn is composed of $N_{\mathrm{E}}$ excitatory (pyramidal) neurons ("E neurons"), that are recurrently connected to an inhibitory interneuron (Fig. 1A). The minicolumns are laterally connected via a sparse, random, plastic connectivity between the E neurons. Each E neuron receives $K_{\mathrm{EE}}$ randomly chosen connections from $E$ neurons in other minicolumns. Initially, these $K_{\mathrm{EE}}$ connections are 'potential' connections. Only during the learning process, these potential connections can turn into effective synaptic connections (see Sec. 2.3).

Feedforward inputs representing the sequence elements are applied to the network in the form of $N_{\text {stim }}$ external spike sources, each being connected to the $\mathrm{E}$ neurons of a random subset of $L$ minicolumns (Fig. 1B).

\section{$2.2 \quad$ Neuron model}

The excitatory neurons are described as multicompartment models comprising a soma and $N_{\mathrm{D}}$ dendritic branches (Fig. 2). Each of the $N_{\mathrm{D}}+1$ compartments is described by a leaky integrate-and-fire (LIF) dynamics [6]. Each dendritic compartment receives excitatory $K_{\mathrm{D}}=K_{\mathrm{EE}} / N_{\mathrm{D}}$ inputs from $\mathrm{E}$ neurons in other minicolumns. Upon threshold crossing, the dendritic compartments generate action potentials ("NMDA spikes") that are propagated to the soma via 
A

B

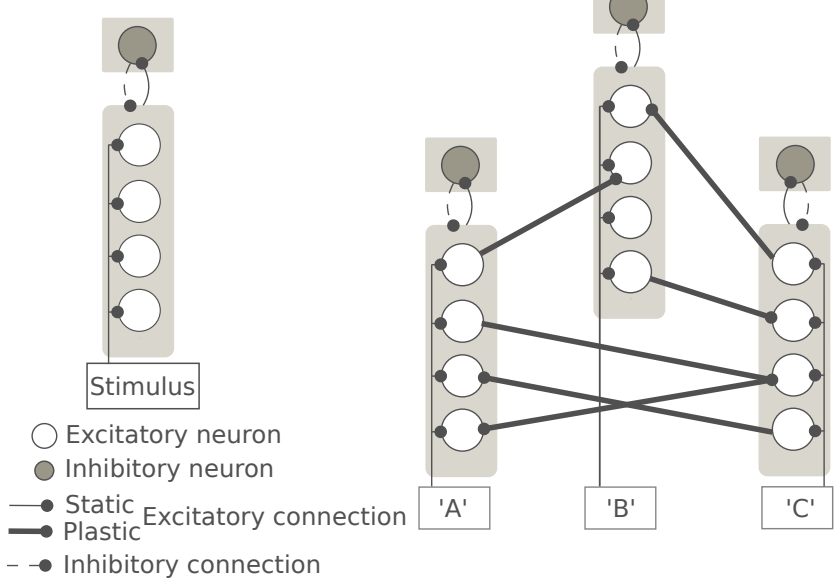

Figure 1: Sketch of the network architecture. A) Each minicolumn is composed of a number of excitatory neurons, which are recurrently connected to an inhibitory neuron, and driven by a feed-forward input (stimulus) representing an element in a sequence. B) The network is composed of a number of minicolumns, each representing an element (e.g. 'A', 'B', 'C') in a sequence. Minicolumns are interconnected via (learned) excitatory connections.

strong but subthreshold weights $J_{\mathrm{SD}}[7,8]$. To enable spike timing dependent plasticity at the dendritic synapses, somatic action potentials are backpropagated to the dendrite via suprathreshold connections with weight $J_{\mathrm{DS}}$ [9-11]. Inhibitory interneurons are described as simple point neuron models with LIF dynamics.

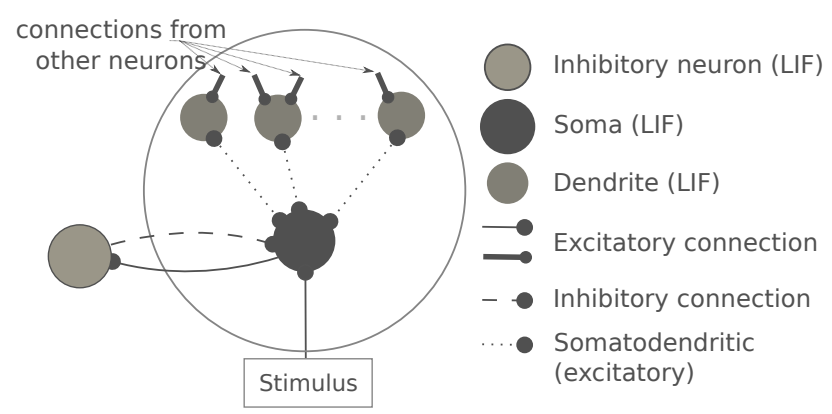

Figure 2: Sketch of the neuron model. Excitatory neurons (large white circle) are composed of a soma and a number of dendritic compartments. Each compartment is modeled as a leaky integrate-and-fire (LIF) unit. The feed-forward input ('stimulus') projects to the soma. Inputs from other minicolumns arrive at the dendritic compartments. Connections between the dendritic compartments and the soma are bidirectional to permit propagation of dendritic spikes to the soma and backpropagation of somatic spikes to the dendrites. Inhibitory (LIF) neurons are communicating with the soma.

\subsection{Synapse models}

For all types of connection in the network, synapses are modeled as exponential currents with amplitude $\hat{I}$, time constant $\tau_{\text {s }}$ and delay $d$. We parameterize the strength of each connection by the weight $J$, which corresponds to the amplitude of the membrane potential deflection caused by a single synaptic event. We distinguish between four types of connections:

EE synapses refer to connections from the somata of E neurons to the dendrites of other E neurons. The strength of these connections is determined by a Hebbian form of structural plasticity: Each EE synapse is characterized by a permanence $P$ and a weight $J_{\mathrm{EE}}$. The permanence models the level of maturity of the synapse. Premature synapses, i.e. synapses where $P$ is below a threshold $\theta_{\mathrm{P}}$, are not effective and assigned zero weight $J_{\mathrm{EE}}=0$. If $P>\theta_{P}, J_{\mathrm{EE}}$ is set to a fixed value $W$, which is chosen such that the target neuron generates a dendritic spike if $\theta_{\mathrm{E}}$ presynaptic neurons fire collectively. For each postsynaptic (dendritic) spike, the permanence $P$ is updated with an increment $\Delta_{f} P$ or decrement $\Delta_{d} P$ that depends on the difference $t_{\text {post }}-t_{\text {pre }}$ between the timing of pre- and postsynaptic spikes. This form of spike timing dependent structural plasticity mimics the plasticity mechanism used in the original HTM model, and is also known from the neuroscience literature [12]. It promotes the formation of connections between neurons that are consistently activated in a sequence and the pruning of unused connections.

IE synapses represent the connections from the somata of $\mathrm{E}$ neurons to the inhibitory neurons. The weights $J_{\text {IE }}$ are set such that the collective firing of a certain number of $\theta_{\mathrm{I}} \mathrm{E}$ neurons in the minicolumn causes the inhibitory neuron to fire.

EI synapses refer to connections from the inhibitory neurons to the somata of $\mathrm{E}$ neurons in the respective minicolumn with a strong negative weight $J_{\mathrm{EI}}$.

EF synapses characterize connections from the external feedforward input sources to the somata of E neurons with suprathreshold weight $J_{\text {EF }}$.

Apart from the EE connections, all synaptic weights are constant (static).

\subsection{Simulation details}

The network simulations are performed in NEST version 2.16.0 [13]. Network states are synchronously updated using exact integration of the system dynamics on a discrete time grid with step size $\delta t$ [14]. For every network realization, the initial permanences and membrane potentials (at time 0 ) are drawn randomly and independently from uniform distributions in the interval $\left[P_{0}, \theta_{\mathrm{P}}\right)$ and $\left[V_{r}, \theta\right)$, respectively.

\section{RESULTS}

\subsection{Sequence learning and prediction in networks of spiking neurons}

All neurons in a given minicolumn share the same feedforward input. Before learning or at the beginning of a new sequence, a feedforward spike will cause all neurons in the respective minicolumn to fire (Fig. 3A). In the HTM model, this is referred to as columnar bursting. If the number of inputs to a dendritic branch from a specific presynaptic minicolumn exceeds a threshold $\theta_{\mathrm{E}}$, the 
simultaneous activation of these presynaptic neurons results in a dendritic spike, followed by a strong depolarization of the soma which corresponds to the "predictive" state (Fig. 3B). Predictive neurons receiving a feedforward input will fire earlier as compared to nonpredictive neurons (Fig. 3C). The advanced spike of the predictive neurons will initiate a fast and strong inhibitory feedback to the entire minicolumn, ultimately suppressing the firing of the nonpredictive neurons.

As described in Sec. 2.3, the EE connections are subject to spike timing dependent structural plasticity. Repetitive sequential activation of feedforward inputs (characters) during the learning therefore leads to the formation of a connectivity structure that corresponds to an imprint of the presented sequences in the sense that the activation of a certain minicolumn by a feedforward input will generate dendritic spikes, i.e. a "prediction", in a specific subset of neurons in the minicolumns representing the next sequence element.

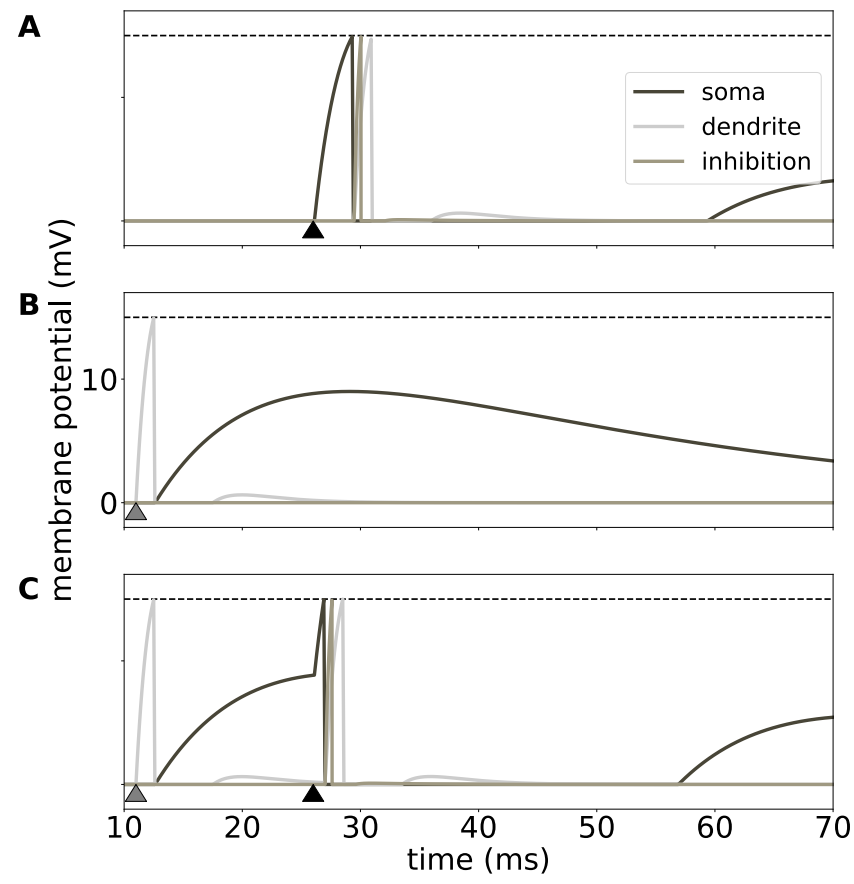

Figure 3: Membrane-potential responses to a feedforward input (black arrow; A), a suprathreshold dendritic input (gray arrow; B), and a combination of feedforward and dendritic input (C). Bars mark spike times. A dendritic spike preceding the feedforward input (as in C) can speed up somatic, and hence, inhibitory firing, provided the time interval between the dendritic spike and the feedforward input is in the right range (compare somatic and inhibitory spikes in $\mathrm{A}$ and $\mathrm{C}$ ). The advanced firing of the inhibitory neuron suppresses somatic spiking in neurons of the same minicolumn that have not received suprathreshold dendritic activation. Here, we used an E neuron with $N_{\mathrm{D}}=1$ dendritic compartment. The some of $\mathrm{E}$ neuron is connected to an inhibitory neuron.

\subsection{Sequence prediction performance}

To test the sequence prediction performance, we repetitively stimulate the network with batches (trials) of $S=2$ sequences, each sequence consisting of $C=10$ characters randomly chosen (with replacement) from an alphabet of length $A=10$. Within a sequence, the same character can occur multiple times. Each batch consists of the same set of sequences with identical order.

The prediction error is continuously monitored and assessed for every batch as follows: For each presentation of a character $q$ (feedforward input) at time $t_{0}$, find all dendrites that are firing in the time interval $\left(t_{0}-\Delta T, t_{0}\right)$, with $\Delta T$ being the inter-stimulus timing. If a minicolumn contains at least $\theta_{\mathrm{I}} \mathrm{E}$ neurons with firing dendrites, this minicolumn is considered as active. The activation of minicolumns is encoded in a binary vector $z$ with $z_{i}=1(i=1, \ldots, M)$ if the $i$ th minicolumn is active, and $z_{i}=0$ else. The prediction error is computed as the Euclidean distance between $z$ and the binary target vector $\boldsymbol{t}$ representing the pattern of feedforward inputs for each character $q$. At the end of each batch, we compute the total prediction error by averaging over all characters within this batch. When assessing the prediction error, we discard the first character in each sequence.

After each new network instantiation, the initial normalized prediction error is at $100 \%$. With increasing number of training episodes (presentation of a batch), the prediction error decreases as the network explores and learns the sequences, and saturates after about 300 episodes (Fig. 4). Note that the model accounts for context dependence: prediction of the next character depends not only on the current sequence element, but on all preceding characters within this sequence. For the first character in a sequence, the context is not defined. As a result, the network predicts all possible subsequent characters from all contexts (i.e. all sequences, where the same character occurs), thereby leading to a residual error, here slighly below $20 \%$.

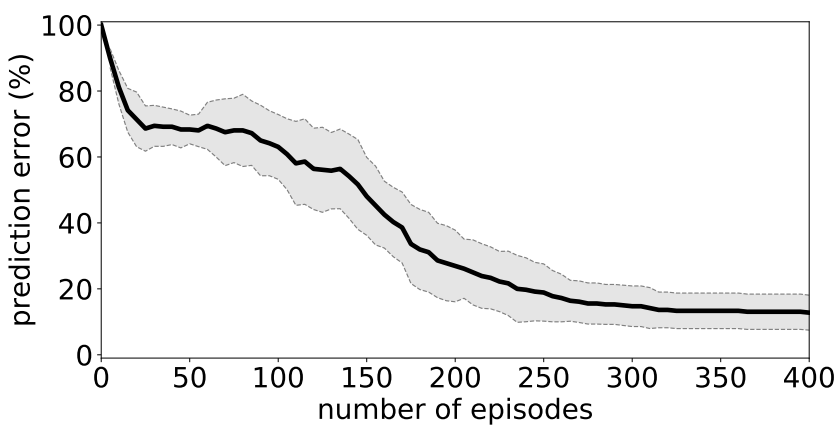

Figure 4: Dependence of the normalized sequence prediction error on the number of training episodes. Solid and dashed curves represent mean \pm standard deviation of the prediction error across 15 different network realizations. Parameters: number of minicolumns $M=10$, number of neurons per minicolumn $N_{\mathrm{E}}=30$, number of dendrites per neuron $N_{\mathrm{D}}=1$, number of synapses per dendrite $K_{\mathrm{D}}=180$, number of sequences per batch $S=2$, number of characters per sequence $C=10$, length of alphabet $A=10$, inter-stimulus interval $\Delta T=80 \mathrm{~ms}$, prediction error assessment for every 4th batch (see Appendix B for remaining parameters).

\subsection{Role of the stimulus timing}

In general, the timing of the feedforward inputs affects the dynamics of the network in two respects: first, reliable predictions of sequence elements can only be made if the time interval $\Delta T$ between two 
consecutive stimulus presentations is such that the second input sufficiently coincides with the somatic depolarization caused by the dendritic spike triggered by the first stimulus (Fig. 3C). Second, only if the time difference between consecutive stimulus presentations matches the potentiation window of the spike timing dependent structural plasticity rule, the network can learn the sequences. Here, we restrict our investigation to the first factor. The second factor is removed by adjusting the potentiation window $\left[\Delta T-\Delta t_{\mathrm{P}}, \Delta T+\Delta t_{\mathrm{P}}\right]$ of the plasticity rule when varying $\Delta T$.

The model predicts both an upper and a lower bound for the optimal inter-stimulus interval $\Delta T$ (Fig. 5). For our choice of network parameters, the optimal inter-stimulus intervals are in the range of about $20-110 \mathrm{~ms}$. For inter-stimulus interval outside this range, the feedforward spike misses the somatic response to the dendritic spike, ultimately leading to a columnar burst. The optimal range of inter-stimulus intervals is determined by the neuronal and synaptic time constants, in particular by the width of the somatic postsynaptic potential caused by a dendritic spike, but also by the strength $J_{S D}$ of the somatodendritic coupling.

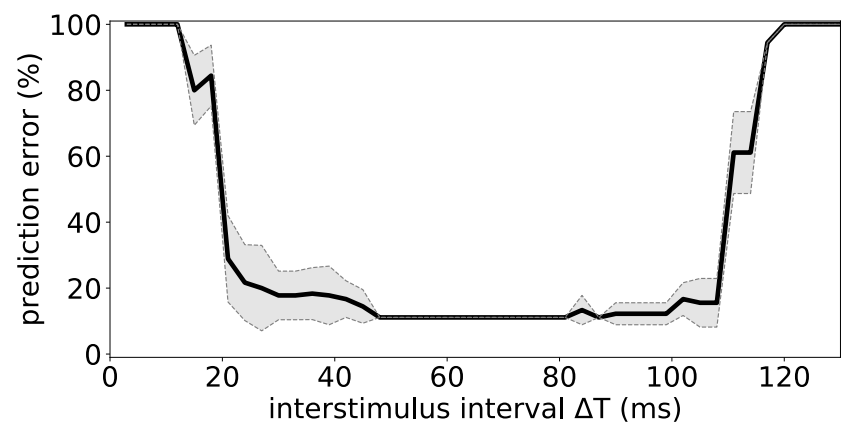

Figure 5: Dependence of the normalized sequence prediction error on the interstimulus interval $\Delta T$ (after 400 training episodes). Same parameters as in Fig. 4.

\section{DISCUSSION}

In the context of our study, the sequence processing speed is determined by the time interval $\Delta T$ between subsequent presentations of sequence elements that guarantee a successful prediction, i.e. a coincidence between the feedforward input and the somatic depolarization caused by dendritic spikes. Our model demonstrates that the optimal range of inter-stimulus intervals is solely determined by low-level neuronal and synaptic parameters, but not by the task complexity such as the sequence or alphabet length, the number of learned sequences, or the ambiguity level [5].

Our model suggests a number of biological mechanisms by which the processing speed could be modulated. Candidates are variations in synaptic or membrane time constants, e.g. controlled by the level of synaptic background input, or a modulation in the effective strength of feedforward inputs or somatodendritic coupling, e.g. through synaptic or intrinsic plasticity.

Note that an HTM implementation based on spiking neurons with continuous-time dynamics was already presented in [15]. This implementation mainly served the purpose of porting the HTM model to an analog-digital neuromorphic hardware system, and differs from our model in several respects. The study did not address the role of neuronal parameters for the sequence processing speed.

The network model underlying our study is still very simplistic. Lateral inhibition within a minicolumn, for example, is mediated by a single interneuron with unrealistically strong and fast connections to and from the pool of excitatory neurons. In future versions, we will replace this interneuron by a recurrently connected network of inhibitory neurons [16], thereby permitting more realistic weights and simultaneously speeding up the interaction between inhibitory and excitatory cells by virtue of the fast-tracking property of such networks [17]. Another limiting factor of the current implementation is the network size: the number of minicolumns, the number of neurons within minicolumns, the number of dendritic branches per neuron, as well as the number of synapses per neuron are far from realistic [5]. The number of sequences that can be successfully learned in this network is hence small. Note, however, that high network capacity is not the central aim of this study. The model is minimalistic on purpose and serves as a means to understand what network characteristics determine the sequence processing speed. In future work, however, we aim at upscaling the network, thereby improving its sequence processing capacity and performance.

\section{ACKNOWLEDGMENTS}

This project was funded by the Helmholtz Association Initiative and Networking Fund (project number SO-092, Advanced Computing Architectures), and the European Union's Horizon 2020 Framework Programme for Research and Innovation under the Specific Grant Agreement No. 785907 (Human Brain Project SGA2).

\section{REFERENCES}

[1] Lashley, K. S. (1951). The problem of serial order in behavior, Volume 21. BobbsMerrill.

[2] Hawkins, J., \& Blakeslee, S. (2007). On intelligence: How a new understanding of the brain will lead to the creation of truly intelligent machines. Macmillan.

[3] Gavornik, J. P., \& Bear, M. F. (2014). Learned spatiotemporal sequence recognition and prediction in primary visual cortex. Nature neuroscience 17(5), 732.

[4] Hawkins, J., Ahmad, S., \& Dubinsky, D. (2011). Cortical learning algorithm and hierarchical temporal memory. Numenta Whitepaper, 1-68.

[5] Hawkins, J., \& Ahmad, S. (2016). Why neurons have thousands of synapses, a theory of sequence memory in neocortex. Frontiers in Neural Circuits 10.

[6] Lapicque, L. (1907). Recherches quantitatives sur l'excitation electrique des nerfs traitee comme une polarization. 7. Physiol. Pathol. Gen. 9, 620-635.

[7] Antic, S. D., Zhou, W.-L., Moore, A. R., Short, S. M., \& Ikonomu, K. D. (2010). The decade of the dendritic nmda spike. Fournal of neuroscience research 88(14), 2991-3001.

[8] Major, G., Larkum, M., \& Schiller, J. (2013). Active properties of neocortical pyramidal neuron dendrites. Annu. Rev. Neurosci. 36, 1-24.

[9] Stuart, G. J., \& Häusser, M. (2001). Dendritic coincidence detection of epsps and action potentials. Nature neuroscience 4(1), 63.

[10] Chklovskii, D., Mel, B., \& Svoboda, K. (2004). Cortical rewiring and information storage. Nature 431, 782-788.

[11] Losonczy, A., Makara, J. K., \& Magee, J. C. (2008). Compartmentalized dendritic plasticity and input feature storage in neurons. Nature 452(7186), 436.

[12] Deger, M., Helias, M., Rotter, S., \& Diesmann, M. (2012). Spike-timing dependence of structural plasticity explains cooperative synapse formation in the neocortex. PLoS Comput. Biol. 8(9), e1002689.

[13] Linssen, C., Lepperød, M. E., Mitchell, J., Pronold, J., Eppler, J. M., Keup, C., Peyser, A., Kunkel, S., Weidel, P., Nodem, Y., Terhorst, D., Deepu, R., Deger, M., Hahne, J., Sinha, A., Antonietti, A., Schmidt, M., Paz, L., Garrido, J., Ippen, T., Riquelme, L., Serenko, A., Kühn, T., Kitayama, I., Mørk, H., Spreizer, S., Jordan, J., Krishnan, J., Senden, M., Hagen, E., Shusharin, A., Vennemo, S. B., Rodarie, D., Morrison, A., Graber, S., Schuecker, J., Diaz, S., Zajzon, B., \& Plesser, H. E. (2018). NEST 2.16.0. [14] Rotter, S., \& Diesmann, M. (1999). Exact digital simulation of time-invariant linear systems with applications to neuronal modeling. Biol. Cybern. 81(5-6), 381-402. [15] Billaudelle, S., \& Ahmad, S. (2015). Porting htm models to the heidelberg neuromorphic computing platform. arXiv preprint arXiv:1505.02142. 
[16] Hu, H., Gan, J., \& Jonas, P. (2014). Fast-spiking, parvalbumin+ gabaergic interneurons: From cellular design to microcircuit function. Science 345(6196), 1255263.

[17] van Vreeswijk, C., \& Sompolinsky, H. (1998). Chaotic balanced state in a model of cortical circuits. Neural Comput. 10(6), 1321-1371. 


\section{A NETWORK MODEL}

\begin{tabular}{|c|c|c|}
\hline \multicolumn{3}{|c|}{ Summary } \\
\hline Populations & \multicolumn{2}{|c|}{ ensemble of minicolumns, each composed of an excitatory and an inhibitory subpopulation } \\
\hline Connectivity & \multicolumn{2}{|c|}{$\begin{array}{l}\text { - sparse random connectivity between excitatory neurons subject to spike-timing- } \\
\text { dependent structural plasticity } \\
\text { - local recurrent connectivity between excitatory and inhibitory neurons (static) }\end{array}$} \\
\hline Neuron model & \multicolumn{2}{|c|}{$\begin{array}{l}\text { - excitatory neurons: multi-compartment model with one soma compartment and one } \\
\text { compartment per dendritic branch, each compartment modeled by leaky integrate-and- } \\
\text { fire (LIF) dynamics } \\
\text { - inhibitory neurons: point neuron (LIF) }\end{array}$} \\
\hline Synapse model & \multicolumn{2}{|c|}{ additive (linear) exponential postsynaptic currents (PSCs) } \\
\hline Plasticity & \multicolumn{2}{|c|}{ spike-timing-dependent structural plasticity in excitatory-to-excitatory connections } \\
\hline Input & \multicolumn{2}{|c|}{ external spike sources, connected to excitatory neurons } \\
\hline \multicolumn{3}{|c|}{ Populations } \\
\hline \multicolumn{3}{|c|}{ Ensemble of minicolumns $\{i \mid i=1, \ldots, M\}$} \\
\hline Name & Elements & Size \\
\hline $\mathcal{E}_{i}$ & excitatory neurons in minicolumn $i$ & $n_{\mathrm{E}}$ \\
\hline $\mathcal{I}_{i}$ & inhibitory neurons in minicolumn $i$ & $n_{\mathrm{I}}$ \\
\hline & \multicolumn{2}{|c|}{ Connectivity } \\
\hline Source & \multicolumn{2}{|l|}{ Pattern } \\
\hline \begin{tabular}{l|l}
$\mathcal{E}_{i}(\forall i)$ & $\mathcal{E}_{j}(\forall j)$ \\
\end{tabular} & \multicolumn{2}{|c|}{ random, fixed in-degrees $K_{\mathrm{EE}}$ and delays $d_{\mathrm{EE}}$, plastic weights $J_{\mathrm{EE}, j i}$ (EE connections) } \\
\hline $\mathcal{E}_{i}(\forall i)$ & \multicolumn{2}{|c|}{ all-to-all, fixed delays $d_{\mathrm{IE}}$, fixed weights $J_{\mathrm{IE}}$ (suprathreshold) (IE connections) } \\
\hline \begin{tabular}{l|l}
$\mathcal{I}_{i}(\forall i)$ & $\mathcal{E}_{i}(\forall i)$ \\
\end{tabular} & \multicolumn{2}{|c|}{ all-to-all, fixed delays $d_{\mathrm{EI}}$, fixed weights $J_{\mathrm{EI}}$ (EI connections) } \\
\hline \begin{tabular}{l|l}
$\mathcal{I}_{i}(\forall i)$ & $\mathcal{I}_{i}(\forall i)$ \\
\end{tabular} & \multicolumn{2}{|c|}{ none (II connections) } \\
\hline \multicolumn{3}{|c|}{ no self-connections (“autapses"), no multiple connections (“multapses") } \\
\hline \multicolumn{3}{|c|}{$\begin{array}{ll} & \text { Neuron } \\
\end{array}$} \\
\hline Excitatory neurons & \multicolumn{2}{|c|}{$\begin{array}{l}\text { multi-compartment model with one soma compartment and } N_{\mathrm{D}} \text { dendritic branches } \\
\text { - } \text { soma compartment: } \\
\text { - leaky-integrate-and-fire (LIF) dynamics (see below) } \\
\text { - dendritic compartments: } \\
\text { - LIF dynamics (see below) } \\
\text { - } K_{\mathrm{D}} \text { (excitatory) synapses per dendritic branch }\end{array}$} \\
\hline Inhibitory neurons & \multicolumn{2}{|l|}{ point neuron (LIF) } \\
\hline $\begin{array}{l}\text { Dynamics of single } \\
\text { compartments (LIF) }\end{array}$ & \multicolumn{2}{|c|}{$\begin{array}{l}\text { dynamics of membrane potential } V_{c}(t) \text { of compartment } c \text { : } \\
\text { - spike emission at time } t_{k}^{c} \text { if } V_{c}\left(t_{k}^{c}\right) \geq \theta \\
\text { - subthreshold dynamics: } \tau_{\mathrm{m}} \dot{V}_{c}=-V_{c}+R_{\mathrm{m}} I_{c}(t) \quad \forall k, \forall t \notin\left[t_{k}^{c}, t_{k}^{c}+\tau_{\mathrm{ref}}\right) \\
\text { - reset and refractoriness: } V_{c}(t)=V_{\mathrm{r}} \quad \forall k, \forall t \in\left(t_{k}^{c}, t_{k}^{c}+\tau_{\mathrm{ref}}\right]\end{array}$} \\
\hline $\begin{array}{l}\text { Coupling between } \\
\text { compartments of } \\
\text { excitatory neurons }\end{array}$ & \multicolumn{2}{|c|}{$\begin{array}{l}\text { - pulse-coupled interaction (spikes) } \\
\text { - dendrite } \rightarrow \text { soma coupling: linear exponential post-synaptic current, fixed weight } J_{\mathrm{SD}} \\
\text { (PSP amplitude), time constant } \tau_{\mathrm{SD}} \text {, delay } d_{\mathrm{SD}} \\
\text { - soma } \rightarrow \text { dendrite coupling (action-potential backpropagation): linear exponential post- } \\
\text { synaptic current, fixed weight } J_{\mathrm{DS}} \text { (PSP amplitude, suprathreshold), time constant } \tau_{\mathrm{DS}} \text {, } \\
\text { delay } d_{\mathrm{DS}}\end{array}$} \\
\hline
\end{tabular}




\begin{tabular}{|c|c|}
\hline & Synapse \\
\hline Model & $\begin{array}{l}\text { current based synapses with additive exponential post-synaptic currents (PSCs): } \\
I_{i}(t)=\sum_{j=1}^{N} \hat{I}_{i j}\left(\operatorname{PSC} * s_{j}\right)(t) \\
\text { with } \operatorname{PSC}(t)=e^{-t / \tau_{\mathrm{s}}} \Theta(t) \text { and Heaviside function } \Theta(t)= \begin{cases}1 & t \geq 0 \\
0 & \text { else }\end{cases} \\
\curvearrowright \text { post-synaptic potential } \operatorname{PSP}_{i j}(t)=\hat{I}_{i j} \frac{R_{\mathrm{m}} \tau_{\mathrm{s}}}{\tau_{\mathrm{s}}-\tau_{\mathrm{m}}}\left(e^{-t / \tau_{\mathrm{s}}}-e^{-t / \tau_{\mathrm{m}}}\right) \Theta(t)\end{array}$ \\
\hline Plasticity & $\begin{array}{l}\text { spike-timing dependent structural plasticity for EE connections; each synapse described by } \\
\text { permanence } P \text { (maturity) and synaptic weight } J_{\mathrm{EE}} \text {; } \\
\text { update of } P_{i j} \text { and } J_{\mathrm{EE}, i j} \text { for each postsynaptic spike at time } t_{\mathrm{post}} \text { : } \\
\quad \text { if } \Delta T-\Delta t_{\mathrm{P}}<t_{\mathrm{post}}-t_{\mathrm{pre}}<\Delta T+\Delta t_{\mathrm{P}} \text { then } \\
\quad P \leftarrow P+\Delta_{f} P \\
\text { else } \\
\quad P \leftarrow P-\Delta_{d} P \\
\text { end if } \\
\text { if } P>P_{\max } \text { then } \\
\quad P=P_{\max } \\
\text { end if } \\
\text { if } P>P_{\theta} \text { then } \\
\quad J_{\mathrm{EE}} \leftarrow W \\
\text { else } \\
\quad J_{\mathrm{EE}} \leftarrow 0 \\
\text { end if } \\
\text { here, } t_{\mathrm{pre}} \text { denotes the spike timing of the presynaptic neuron. } \Delta T \text { is the interstimulus timing, } \\
\Delta t_{P} \text { the range from } \Delta T \text { at which the permanence is incremented and } \Delta P \text { is the amount used to } \\
\text { increment the permanence. }\end{array}$ \\
\hline
\end{tabular}

- $N_{\text {stim }}$ spike-generating sources, each representing a character in the sequences

- each spike source connected to a random subset of $L$ minicolumns via a synaptic weight $J_{\mathrm{EF}}$ and a delay of $d_{\mathrm{EF}}$

\section{Training}

- repetitive stimulation of network ( $k$ times) using the same batch of data.

- a batch of data contains $\mathrm{S}$ sequences, each sequence is composed of $\mathrm{C}$ characters

\section{Initial conditions}

- for every network realization: initial permanences and membrane potentials (at time 0 ) randomly and independently drawn from uniform distributions in intervals $\left[P_{0}, \theta_{\mathrm{P}}\right)$ and $\left[V_{r}, \theta\right)$, respectively

\section{Simulation details}

- network simulations performed in NEST version 2.16.0 [13]

- synchronous update using exact integration of the system dynamics on a discrete time grid with step size $\Delta t$ [14] 


\section{B PARAMETERS}

\begin{tabular}{|c|c|c|}
\hline Name & Value & Description \\
\hline \multicolumn{3}{|r|}{ Network } \\
\hline$n_{\mathrm{E}}$ & 30 & number of excitatory neurons per minicolumns \\
\hline$n_{\mathrm{I}}$ & 1 & number of inhibitory neurons per minicolumns \\
\hline$M$ & 10 & number of minicolumns \\
\hline \multicolumn{3}{|c|}{ (Potential) Connectivity } \\
\hline$K_{\mathrm{EE}}$ & 180 & number of excitatory inputs per excitatory neuron (EE in-degree) \\
\hline$K_{\mathrm{EI}}$ & 1 & number of inhibitory inputs per excitatory neuron (EI in-degree) \\
\hline$K_{\mathrm{IE}}$ & $n_{\mathrm{E}}$ & number of excitatory inputs per inhibitory neuron (IE in-degree) \\
\hline$K_{\mathrm{II}}$ & 0 & number of inhibitory inputs per inhibitory neuron (II in-degree) \\
\hline \multicolumn{3}{|c|}{ Excitatory neurons } \\
\hline \multicolumn{3}{|r|}{ Soma } \\
\hline$\tau_{\mathrm{m}}$ & $10 \mathrm{~ms}$ & membrane time constant \\
\hline$\tau_{\text {ref }}$ & $30 \mathrm{~ms}$ & absolute refractory period \\
\hline$C_{\mathrm{m}}$ & $250 \mathrm{pF}$ & membrane capacity \\
\hline$V_{\mathrm{r}}$ & $0.0 \mathrm{mV}$ & reset potential \\
\hline$\theta$ & $15 \mathrm{mV}$ & spike threshold \\
\hline \multicolumn{3}{|r|}{ Dendrite } \\
\hline$N_{\mathrm{D}}$ & 1 & number of dendritic branches per excitatory neuron \\
\hline$K_{\mathrm{D}}$ & $180\left(K_{\mathrm{D}}=K_{\mathrm{EE}}\right)$ & number of synapses per dendritic branch \\
\hline$\tau_{\mathrm{m}}$ & $3 \mathrm{~ms}$ & membrane time constant \\
\hline$\tau_{\text {ref }}$ & $2 \mathrm{~ms}$ & absolute refractory period \\
\hline$C_{\mathrm{m}}$ & $250 \mathrm{pF}$ & membrane capacity \\
\hline$V_{\mathrm{r}}$ & $0.0 \mathrm{mV}$ & reset potential \\
\hline$\theta$ & $15 \mathrm{mV}$ & spike threshold \\
\hline \multicolumn{3}{|c|}{ Somato-dendritic coupling } \\
\hline$J_{\mathrm{SD}}$ & $9.0 \mathrm{mV}$ & $\begin{array}{l}\text { dendrite } \rightarrow \text { soma coupling strength (amplitude of PSP evoked } \\
\text { by dendritic spike) }\end{array}$ \\
\hline$J_{\mathrm{DS}}$ & $8.25 \mathrm{mV}$ & $\begin{array}{l}\text { soma } \rightarrow \text { dendrite coupling strength (amplitude of dendritic PSP } \\
\text { evoked by somatic spike) }\end{array}$ \\
\hline$\tau_{\mathrm{SD}}$ & $30 \mathrm{~ms}$ & $\begin{array}{l}\text { time constant of dendrite } \rightarrow \text { soma coupling (post-synaptic cur- } \\
\text { rent) }\end{array}$ \\
\hline$\tau_{\mathrm{DS}}$ & $2 \mathrm{~ms}$ & $\begin{array}{l}\text { time constant of soma } \rightarrow \text { dendrite coupling (post-synaptic cur- } \\
\text { rent) }\end{array}$ \\
\hline$d_{\mathrm{SD}}$ & $0.1 \mathrm{~ms}$ & delay of dendrite $\rightarrow$ soma coupling \\
\hline$d_{\mathrm{DS}}$ & $0.1 \mathrm{~ms}$ & delay of soma $\rightarrow$ dendrite coupling \\
\hline \multicolumn{3}{|c|}{ Inhibitory neurons } \\
\hline$\tau_{\mathrm{m}}$ & $5 \mathrm{~ms}$ & membrane time constant \\
\hline$\tau_{\text {ref }}$ & $2 \mathrm{~ms}$ & absolute refractory period \\
\hline$C_{\mathrm{m}}$ & $250 \mathrm{pF}$ & membrane capacity \\
\hline$V_{\mathrm{r}}$ & $0.0 \mathrm{mV}$ & reset potential \\
\hline$\theta$ & $15 \mathrm{mV}$ & spike threshold \\
\hline
\end{tabular}




\begin{tabular}{|c|c|c|}
\hline Name & Value & Description \\
\hline \multicolumn{3}{|r|}{ Synapse } \\
\hline$J_{\mathrm{EE}, i j}$ & $\{0, W\}$ & $\begin{array}{l}\text { E-neuron } \rightarrow \text { E-neuron connections strength (amplitude of den- } \\
\text { dritic PSP of neuron } i \text { evoked by somatic spike of neuron } j \text { ) }\end{array}$ \\
\hline W & $7.5 \mathrm{mV}$ & $\begin{array}{l}\text { amplitude of PSP evoked by somatic spike in the case of a mature } \\
\text { synapse, } \theta_{\mathrm{E}}=\frac{\theta}{W}=2\end{array}$ \\
\hline$J_{\mathrm{IE}}$ & $3.75 \mathrm{mV}$ & $\begin{array}{l}\text { E-neuron } \rightarrow \text { I-neuron connections strength (amplitude of the } \\
\text { inhibitory neuron } i \text { evoked by somatic spike of neuron } i), \theta_{\mathrm{I}}= \\
\frac{\theta}{J_{\mathrm{IE}}}=4\end{array}$ \\
\hline$J_{\mathrm{EI}}$ & $-22.5 \mathrm{mV}$ & $\begin{array}{l}\text { I-neuron } \rightarrow \text { E-neuron connections strength (amplitude of so- } \\
\text { matic PSP of neuron } i \text { evoked by the inhibitory neuron } i \text { ) }\end{array}$ \\
\hline$\tau_{\mathrm{s}, \mathrm{EE}}$ & $2 \mathrm{~ms}$ & $\begin{array}{l}\text { time constant of E-neuron } \rightarrow \text { E-neuron connection (post- } \\
\text { synaptic current) }\end{array}$ \\
\hline$\tau_{\mathrm{s}, \mathrm{IE}}$ & $0.5 \mathrm{~ms}$ & $\begin{array}{l}\text { time constant of E-neuron } \rightarrow \text { I-neuron connection (post-synaptic } \\
\text { current) }\end{array}$ \\
\hline$\tau_{\mathrm{SEI}}$ & $2 \mathrm{~ms}$ & $\begin{array}{l}\text { time constant of I-neuron } \rightarrow \text { E-neuron connection (post-synaptic } \\
\text { current) }\end{array}$ \\
\hline$d_{\mathrm{EE}}$ & $5 \mathrm{~ms}$ & delay of E-neuron $\rightarrow$ E-neuron connection \\
\hline$d_{\mathrm{IE}}$ & $0.1 \mathrm{~ms}$ & delay of E-neuron $\rightarrow$ I-neuron connection \\
\hline$d_{\mathrm{EI}}$ & $0.1 \mathrm{~ms}$ & delay of I-neuron $\rightarrow$ E-neuron connection \\
\hline \multicolumn{3}{|r|}{ Plasticity } \\
\hline$P_{i j}$ & {$\left[P_{\min }, P_{\theta}\right]$} & synaptic permanence \\
\hline$\Delta_{f} P$ & 3 & permanence increment \\
\hline$\Delta_{d} P$ & 1 & permanence decrement \\
\hline$W$ & $9.0 \mathrm{mV}$ & weight $\left(J_{\mathrm{EE}}\right)$ of mature synapses \\
\hline$P_{\theta}$ & 2300 & synapse maturity threshold \\
\hline$P_{\max }$ & 4000 & upper permanence bound \\
\hline$P_{\min }$ & 2000 & lower permanence bound \\
\hline$\Delta t_{\mathrm{P}}$ & 5 & interval for synaptic increment \\
\hline \multicolumn{3}{|r|}{ Input } \\
\hline$N_{\text {stim }}$ & 10 & number of external spike sources \\
\hline$L$ & 1 & number of minicolumns connected to the same spike source \\
\hline $\mathrm{S}$ & 2 & number of sequences per batch \\
\hline $\mathrm{C}$ & 10 & number of characters per sequence \\
\hline A & 10 & length of the total alphabet \\
\hline$J_{\mathrm{EF}}$ & $16.5 \mathrm{mV}$ & $\begin{array}{l}\text { Feedforward input } \rightarrow \text { E-neuron connections strength (amplitude } \\
\text { of somatic PSP of neuron } i \text { evoked by feedforward spike) }\end{array}$ \\
\hline$\tau_{\mathrm{s}, \mathrm{EF}}$ & $3.16 \mathrm{~ms}$ & $\begin{array}{l}\text { time constant of Feedforward input } \rightarrow \text { E-neuron connection } \\
\text { (post-synaptic current) }\end{array}$ \\
\hline$d_{\mathrm{EF}}$ & $0.1 \mathrm{~ms}$ & delay of Feedforward input $\rightarrow$ E-neuron connection \\
\hline \multicolumn{3}{|r|}{ Simulation } \\
\hline$\Delta T$ & {$[3,180] \mathrm{ms}$} & interstimulus timing \\
\hline$\Delta t$ & $0.1 \mathrm{~ms}$ & time resolution \\
\hline$k$ & 400 & training steps \\
\hline
\end{tabular}

Table 2: Network and simulation parameters 